\title{
Beneficios da aromaterapia com óleo essencial de lavanda nas diferentes fases da vida da mulher
}

\author{
Benefits of aromatherapy with lavender essential oil at different \\ stages of woman's life
}

Recebido em: $12 / 08 / 2021$

Aceito em: $01 / 12 / 2021$

Vitoria Marti GOMES; Maria Aparecida NICOLETTI

Departamento de Farmácia, Faculdade de Ciências Farmacêuticas, Universidade de

São Paulo. Av. Professor Lineu Prestes, 580, CEP 05508-900. São Paulo, SP, Brasil.

E-mail:vitoria.marti76@gmail.com

\section{ABSTRACT}

Throughout their lives, women are affected by symptoms arising from the most diverse reproductive stages they live, starting from youth and extending to older ages, often presenting symptoms that incapacitate them to exercise their activities, affecting their autonomy and also their social visibility. Aromatherapy is a practice that uses the inhalation of essential oils - volatile concentrates extracted from vegetables - in order to improve physical and mental health and also well-being of the user. This work will address the use of aromatherapy with Lavender essential oil as an Integrative and Complementary treatment in relief of symptoms experienced by these women. Therefore, the aim of this study was to demonstrate if the practice of aromatherapy with Lavender essential oil could bring benefits to the women's physical and mental health. Thus, a narrative bibliographic review of the last 10 years was performed, with articles in Portuguese and English languages obtained from the US National Library of Medicine - National Institute of Health (Pubmed) and Google ${ }^{\circledR}$ Scholar databases. Some informations was also obtained from websites of national, international, public and private institutions. All articles selected within the proposed inclusion criteria demonstrated positive results by use of lavender aromatherapy at least one of the symptoms tested in clinical trials, showing that this therapy is able to mitigate symptoms experienced by women.

Keywords: aromatherapy; lavender; womens's health.

\section{RESUMO}

Durante toda a vida, as mulheres são afetadas por sintomas decorrentes das mais diversas fases reprodutivas que vivem, se iniciando desde a juventude podendo se estender até idades mais avançadas, apresentando, muitas vezes, sintomas que as incapacitam de exercerem suas atividades, afetando assim sua autonomia e sua visibilidade social. A aromaterapia é uma prática onde se utiliza a inalação de óleos essenciais - concentrados voláteis extraídos de vegetais - com o objetivo de melhorar a saúde física, mental e o bem-estar do usuário. O trabalho abordará a utilização da aromaterapia com óleo essencial de Lavanda como uma Prática Integrativa e Complementar para o tratamento e alívio dos sintomas vividos 
por essas mulheres. Portanto, o objetivo deste trabalho foi demonstrar se a prática da aromaterapia com óleo essencial de Lavanda poderia ou não trazer benefícios à saúde física e mental das mulheres. Assim, foi realizada uma revisão bibliográfica do tipo narrativa dos últimos 10 anos, com artigos em idiomas português e inglês obtidos pelas bases de dados US National Library of Medicine - National Institute of Health (PubMed), Biblioteca Virtual em Saúde (Literatura Latino-Americana e do Caribe em Ciências da Saúde - LILACS) e Web of Science. Também foram utilizadas informações em relatórios, publicações de sites de instituições nacionais, internacionais, públicas e privadas. Todos os artigos selecionados dentro dos critérios de inclusão propostos apresentaram resultados positivos com relação à utilização da aromaterapia com óleo essencial de lavanda em, ao menos, uma das esferas de sintomas testadas nos ensaios clínicos, demonstrando que essa terapia é capaz de amenizar sintomas de diversas fases vivenciadas pelas mulheres.

Palavras-chave: aromaterapia; lavanda; saúde da mulher

\section{INTRODUÇÃO}

Ao longo de suas trajetórias, as mulheres procuram elevar sua autoestima e singularidades. Durante suas interações com outros indivíduos em seus cotidianos, se revelam, amam, sofrem, são felizes e se transformam. O relacionamento conjugal, por longo tempo, foi um fator importante para o viver saudável, sendo essencial para o processo de socialização da mulher, formação de sua identidade e sentimento de pertencimento a um grupo social. Para as mulheres, a busca da autonomia e dos direitos têm sido primordiais para fortalecer o seu papel social, a vivência plena da cidadania e a sua visibilidade na sociedade (1).

Durante toda a vida, as mulheres são afetadas por sintomas decorrentes das mais diversas fases reprodutivas que vivem, tendo seu início ainda na puberdade com o aparecimento da menarca (primeiro ciclo menstrual) e, frequentemente, da dismenorreia (cólica menstrual) que acompanha as mulheres durante toda a vida fértil causando dores que, muitas vezes, as incapacitam de exercerem suas funções sociais e profissionais.

Um estudo mostrou que $71 \%$ das mulheres com média de idade de 22 anos sofriam de dismenorreia e que, destas, $42 \%$ afirmavam já ter faltado a algum compromisso devido às dores. Segundo as mulheres participantes do estudo, a vitalidade foi o domínio mais afetado nessas condições, interferindo negativamente em suas atividades cotidianas e qualidade de vida durante esse período (2).

Ainda com relação ao período menstrual, é importante citar o desenvolvimento da síndrome pré-menstrual, que afeta física e emocionalmente algumas mulheres nos dias que antecedem a menstruação. Um estudo revelou que mulheres que apresentavam essa síndrome sofriam com mamas inchadas e doloridas, cólicas menstruais, impaciência, quietude e respostas hostis às pessoas, interferindo em suas relações interpessoais (3).

Outro momento de grande impacto na vida reprodutiva de uma mulher é a gestação, onde a mulher passa por diversas mudanças físicas e emocionais e tende a responder a esta nova fase de acordo com suas características pessoais e a sua habilidade de solicitar e aceitar apoio de outras pessoas, bem como à presença de uma rede de apoio disponível.

A experiência da maternidade envolve, pelo menos em alguns momentos, situações potencialmente estressantes e debilitantes ao longo da gestação (4). O parto e o puerpério trazem ainda mais desafios para as mulheres, que vivenciam uma série de mudanças no período do puerpério, desde a considerável diminuição das horas de sono, até a perda de sua autonomia, trazendo uma série de sentimentos novos, que passam a participar de suas tomadas de decisão (5). Além disso, uma situação infelizmente bastante comum é o desenvolvimento da depressão pós-parto, 
que possui etiologia multifatorial, atingindo um número significativo de mulheres durante esse período (6).

A fase que encerra a vida fértil de uma mulher é marcada pela última menstruação, a menopausa, trazendo, novamente, inúmeros desafios e mudanças em sua vida. Observou-se uma associação entre maior intensidade de sintomas climatéricos (fogachos, cansaço, alterações de humor, diminuição do desejo sexual) e desenvolvimento de sintomas de depressão e ansiedade, além da autopercepção de estado de saúde ruim ou péssima, que pode ser causada tanto pelo efeito prejudicial de um pior estado psicológico sobre os sintomas da menopausa quanto pelo efeito negativo dos sintomas climatéricos (7).

Assim, as fases reprodutivas vivenciadas pelas mulheres desencadeiam sintomas físicos e mentais que muitas vezes requerem tratamento para sua participação em seu ciclo social/familiar/profissional e para exercerem suas funções com maior qualidade de vida. Usualmente, a prática terapêutica mais utilizada nesses casos é realizada com medicamentos tradicionais.

A aromaterapia é classificada como uma prática terapêutica participante das Práticas Integrativas e Complementares (PIC). As PIC surgiram a partir de abordagens vindas do Departamento de Medicina Tradicional, criado pela Organização Mundial da Saúde (OMS) em 1972 (8).

Buscando uma política pública permanente que abordasse de forma amplificada o processo saúde-doença e a promoção global do cuidado humano, desenvolveu-se, em 2006, um documento normatizador para institucionalizar as experiências com essas práticas na rede pública e induzir políticas, programas e legislação nas três instâncias de governo: a Política Nacional de Práticas Integrativas e Complementares em Saúde (PNPIC), tornando assim as PIC integradas oficialmente ao Sistema Único de Saúde (SUS) (9).

De acordo com o Ministério da Saúde, as PIC são definidas como tratamentos que utilizam recursos terapêuticos baseados em conhecimentos tradicionais, voltados para prevenção de doenças, podendo ser usadas também como tratamento paliativo em caso de determinadas doenças crônicas. Promovem a estimulação da promo- ção, manutenção e recuperação da saúde de um indivíduo com ênfase na escuta acolhedora, no desenvolvimento do vínculo terapêutico e na integração do ser humano com o meio ambiente e a sociedade, sempre atendendo o paciente em sua singularidade, complexidade e integralidade. Exerce uma cultura de cuidado, fortalecendo o vínculo terapeuta-paciente, o empoderamento do indivíduo e o seu protagonismo no processo de cura (10). É importante ressaltar que as PIC não substituem ou concorrem com qualquer tratamento convencional feito com medicamentos cientificamente comprovados, e sim possibilitam um olhar integrativo na saúde, complementando o tratamento de acordo com as necessidades de cada caso (8).

A aromaterapia é definida como a prática terapêutica que utiliza, intencionalmente, óleos essenciais (concentrados voláteis extraídos de vegetais) buscando promover ou melhorar a saúde física e mental, o bem-estar e a higiene (11). A prática começou a ser estudada na década de 20 , quando um perfumista francês observou melhora da dor em um ferimento após utilizar óleo de lavanda no local, instituindo o termo "aromaterapia”, como é conhecida atualmente (12).

No Brasil, a aromaterapia é conhecida como prática integrativa e complementar, utilizada individualmente ou de forma coletiva em diversas áreas da saúde, visando reequilibrar física e emocionalmente e intervir de forma positiva nos tratamentos realizados pelos pacientes (11). Um dos primeiros passos para a promoção dessa prática em território nacional foi a fundação da Associação Brasileira de Aromaterapia e Aromatologia (ABRAROMA) em 11 de setembro de 1997, sendo grande responsável pelo aprimoramento e fortalecimento da utilização dos óleos essenciais na saúde (13). A prática da aromaterapia, de acordo com a publicação no Diário Oficial da União, foi incorporada à PNPIC em 2018, tornando-se então oficialmente integrada e disponibilizada pelo SUS (11).

As formas de aplicação mais utilizadas na aromaterapia são a inalação, a compressa, a pulverização/difusão aérea, o banho e a massagem. Estudos indicam que na administração via inalatória, as moléculas dos óleos essenciais podem 
ser capazes de estimular os nervos olfativos (diretamente ligados ao Sistema Límbico), liberando neurotransmissores associados à função analgésica e sensação de bem-estar (14).

O óleo essencial tema desta revisão e um dos mais utilizados na aromaterapia, é o óleo de Lavandula sp., que é extraído das flores frescas ou secas de diferentes espécies da planta por meio de destilação a vapor (15) e sua composição apresenta cariofileno, limoneno, pineno, acetato de lavandulina, cineol, borneol, lavandulol, geraniol, linalol e acetato de linalila $(16,17)$. Normalmente, dentro deste gênero, a espécie mais utilizada na produção de óleos com fins medicinais é a Lavandula angustifolia Mill (L. angustifolia).

Considerando o cenário de situações desgastantes para as mulheres, pretende-se verificar neste trabalho se a utilização de terapias não farmacológicas, como a aromaterapia com óleos essenciais, pode contribuir ou não para a diminuição dos sintomas e melhorar a qualidade de vida das mulheres nessas situações.

\section{MÉTODO}

Foi realizada uma revisão bibliográfica narrativa dos últimos 10 anos utilizando as bases de dados US National Library of Medicine - National Institutes of Health (PubMed), Biblioteca Virtual em Saúde (Literatura Latino-Americana e do Caribe em Ciências da Saúde - LILACS) e Web of Science. Os termos "aromaterapia", "lavanda", "dismenorreia", "parto", "pós-parto", "menopausa", "depressão" serão utilizados como palavraschave para a realização da busca.

Os critérios de inclusão foram artigos científicos na íntegra, nos idiomas português e inglês, com espaço amostral de, no mínimo, 95 participantes nos estudos clínicos e que focavam os resultados encontrados em pesquisas de utilização da aromaterapia com óleo essencial de Lavanda, sendo identificados inicialmente pelo título e resumo para, em seguida serem selecionados para serem lidos integralmente. Os critérios de exclusão foram artigos publicados em outros idiomas, fora do período proposto e com $\mathrm{N}$ amostral menor do que o mencionado, além de não estarem disponibilizados na íntegra e de não apresentarem cunho científico em sua estruturação (Figura 1).

Figura 1. Critérios de inclusão e exclusão adotados na busca bibliográfica sobre o tema abordado.

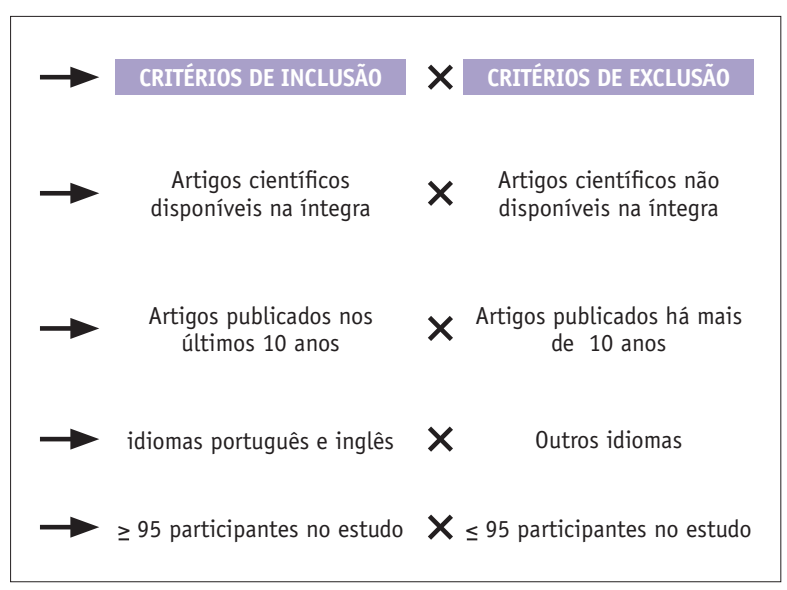

\section{RESULTADOS E DISCUSSÃO}

Os resultados encontrados estão apresentados nos Quadros 1 a 3, divididos em categorias que representam as principais fases de vida das mulheres ao longo de suas trajetórias. Nos quadros, é possível visualizar para quais sintomas cada artigo avaliou clinicamente os benefícios da utilização de aromaterapia com óleos essenciais de Lavandula sp. É importante ressaltar que em ambas as categorias todos os artigos encontrados, com a margem amostral proposta, tiveram impacto positivo quanto à utilização da aromaterapia em pelo menos uma das vertentes propostas, não havendo viés na escolha dos estudos. 
Quadro 1. Fase Adolescência/Juventude da mulher e a utilização da aromaterapia utilizado óleos essenciais de diferentes espécies de Lavandula sp.

\begin{tabular}{|c|c|}
\hline $\begin{array}{l}\text { Autor: Nikjou R., Karimollahi M., Kazemzadeh R., Rostamnegad M., Moshfegi S., Salehi H } \\
\text { Titulo: The Effect of Lavender Aromatherapy on the Pain Severity of Primary Dysmenorrhea: } \\
\text { A Triple-blind Randomized Clinical Trial } \\
\text { Revista: Annals of Medical \& Health Sciences Research } \\
\text { Ano: } 2016\end{array}$ & $\begin{array}{l}\text { Ensaio clínico com estudantes que possuem dismenorreia } \\
\text { primária para avaliar se a aromaterapia com } L \text {. } \\
\text { angustifolia foi capaz de reduzir as dores da dismenorreia. } \\
\qquad N \text { amostral: } 200 \text { participantes }\end{array}$ \\
\hline $\begin{array}{l}\text { Autor: Dehkordi, Z., Baharanchi F., Bekhradi R } \\
\text { Título: Effect of lavender inhalation on the symptoms of primary dysmenorrhea and the } \\
\text { amount of menstrual bleeding: A randomized clinical trial } \\
\text { Revista: Complementary Therapies in Medicine } \\
\text { Ano: } 2014\end{array}$ & $\begin{array}{l}\text { Ensaio clínico com estudantes que sofrem com sintomas } \\
\text { da dismenorreia primária para avaliar se a aromaterapia } \\
\text { com L. angustifolia foi capaz de reduzir os sintomas bem } \\
\text { como a quantidade de sangue menstrual. } \\
\mathrm{N} \text { amostral: } 96 \text { participantes }\end{array}$ \\
\hline $\begin{array}{l}\text { Autor: Azima,S., Bakhshayesh H., Kaviani M., Abbasnia K., Sayadi M } \\
\text { Título: Comparison of the Effect of Massage Therapy and Isometric Exercises on Primary } \\
\text { Dysmenorrhea: A Randomized Controlled Clinical Trial } \\
\text { Revista: Journal of Pediatric and Adolescent Gynecology } \\
\text { Ano: } 2015\end{array}$ & $\begin{array}{l}\text { Ensaio clínico com estudantes que sofrem com sintomas } \\
\text { da dismenorreia primária para avaliar se a massagem e } \\
\text { consequente aromaterapia com Lavandula sp. foi capaz } \\
\text { de diminuir a intensidade dos sintomas da dismenorreia. } \\
\qquad \mathrm{N} \text { amostral: } 120 \text { participantes }\end{array}$ \\
\hline $\begin{array}{l}\text { Autor: Zayeri, F., Dehkordi Z., Hosseini-Baharanchi } F \\
\text { Título: The clinical efficacy of lavender oil inhalation on intensity of menstrual pain from } \\
\text { primary dysmenorrhea } \\
\text { Revista: Journal of Herbmed Pharmacology } \\
\text { Ano: } 2019\end{array}$ & $\begin{array}{l}\text { Ensaio clínico com estudantes que sofrem de } \\
\text { dismenorreia primária para avaliar se a aromaterapia com } \\
\text { L. angustifolia foi capaz de diminuir a intensidade dos } \\
\text { sintomas da dismenorreia. } \\
\mathrm{N} \text { amostral: } 96 \text { participantes }\end{array}$ \\
\hline
\end{tabular}

Os trabalhos apresentados no Quadro 1 focaram na utilização da aromaterapia para alívio de sintomas enfrentados que se iniciam ainda durante a adolescência, se estendendo durante toda a juventude até chegar à fase adulta, como a dismenorreia (também chamada de cólica menstrual), caracterizada pela dor no baixo ventre durante a menstruação, podendo ser acompanhada de náusea, diarreia, dor de cabeça e, em alguns casos, até desmaio (18).

Os artigos discutiram os benefícios da aromaterapia nas dores da dismenorreia durante o período menstrual, época de extrema importância na vida da mulher, uma vez que estudos demonstraram que $71 \%$ das mulheres com média de idade de 22 anos sofrem de dismenorreia e que, destas, $42 \%$ afirmam já ter faltado a algum compromisso devido às dores, sendo a vitalidade o domínio mais afetado nessas condições, interferindo negativamente em suas atividades cotidianas e qualidade de vida (2).

Em um estudo realizado com 200 estudantes portadoras de dismenorreia primária, foram criados dois grupos - controle e experimental - para testar a eficácia da aromaterapia nas dores do período menstrual. O grupo experimental realizou a inalação de óleo essencial de L. angustifolia (3 gotas em algodão, 1 vez ao dia, por 30 minutos, nos três primeiros dias da menstruação e durante dois ciclos menstruais) e o grupo controle realizou o mesmo procedimento, porém com leite diluído pingado no algodão. No início do ciclo menstrual antes da intervenção e após o fim de cada um dos dois ciclos menstruais com a intervenção realizada, as participantes responderam ao questionário visual analogue scale - escala visual analógica VAS para quantificar os níveis de dor observados (19).

A escala de VAS é um método visual que consiste em uma linha reta onde na extremidade lateral esquerda existe uma imagem de rosto sorridente (representando nenhuma dor) e na extremidade lateral direita uma imagem de rosto em sofrimento (representando o pior nível de dor possível). É uma das escalas mais utilizadas para determinar os níveis de dor e de sintomas dos indivíduos, uma vez que a quantificação numérica se torna mais abstrata para a classificação da dor (20).

No ciclo 0 , ou seja, antes da exposição ao óleo essencial e ao placebo, não houve diferença significativamente estatística nos níveis de dores entres os grupos $(\mathrm{P}=0,72)$. Após a intervenção, o grupo experimental demonstrou diminuição esta- 
tisticamente significativa $(\mathrm{P}<0,001)$ nos níveis de dores das participantes em comparação ao grupo controle (19).

Um estudo realizado com 96 estudantes portadoras de dismenorreia demonstrou resultados positivos quanto à redução das dores, mas não foi observada qualquer comprovação positiva com relação à redução de fluxo sanguíneo menstrual. As participantes foram divididas em dois grupos: controle e experimental (21).

O grupo de intervenção realizou a aromaterapia com óleo essencial de L. angustifolia diluído em óleo de sésamo na proporção de 2:1 e o grupo controle utilizou apenas óleo de sésamo atuando como placebo. As estudantes pingaram 3 gotas da solução nas mãos, esfregaram as mãos e as mantiveram a uma distância de $7-10 \mathrm{~cm}$ do rosto, inalando por 5 minutos. O procedimento foi realizado de 6 em 6 horas ao longo dos três primeiros dias do ciclo menstrual, durante dois ciclos menstruais completos. A avaliação da gravidade dos sintomas foi realizada por meio de um questionário formulado por um pesquisador (21).

O questionário foi desenvolvido através da revisão da literatura relacionada e possui uma escala de 0 a 3 , onde: 0 : nenhum sintoma, 1 : sintomas fracos de dismenorreia, que não afeta as atividades diárias, 2: sintomas fracos de dismenorreia, que afeta as atividades diárias, mas não são debilitantes, 3 : sintomas que tornam a paciente completamente debilitada (21).

Os resultados encontrados demonstraram que houve redução nos sintomas no grupo experimental em comparação ao grupo controle que satisfazem a propriedade das probabilidades proporcionais $(\mathrm{P}<0,001)$ enquanto que as medidas basais da quantidade de sangramento menstrual nos primeiros dias do período menstrual não foram significativas entre os grupos $(\mathrm{P}>0,05)(21)$.

Outro estudo, que retratou sobre as dores da dismenorreia, foi realizado com 96 estudantes portadoras de dismenorreia primária, divididas em dois grupos: controle e experimental. Os métodos utilizados foram os mesmos descritos no ensaio clínico anterior (espécie de Lavandula sp. não especificada), porém neste caso foram avaliados apenas os níveis de dores da dismenorreia (fluxo menstrual não avaliado) e o método de avaliação dos sintomas foi a escala de VAS (22).

No primeiro ciclo menstrual, a média da dor pela escala VAS no nível basal (antes da intervenção) não demonstrou diferença significativa entre os grupos experimental e controle $(\mathrm{P}=0,06)$ e após 1, 2, 4 e 48 horas do início da intervenção, houve diminuição significativa da média de dor no grupo experimental em comparação ao grupo controle $(\mathrm{P}<0,001)$. No segundo ciclo menstrual, foi observada redução significativa na média da dor pela escala VAS ainda no nível basal para o grupo experimental em relação ao controle $(\mathrm{P}=0,024)$ e após $1,2,4$ e 48 horas do início da intervenção esse valor se fez ainda mais notável $(\mathrm{P}<0,001)(22)$.

Finalizando o tema da dismenorreia, foi realizado um estudo com 120 estudantes portadoras de dismenorreia primária, divididas em três grupos: experimental com aromaterapia de lavanda; experimental com exercícios isométricos e controle. O grupo experimental de interesse neste trabalho aplicou óleo essencial de L. angustifolia na parte superior da sínfise púbica e umbilical, onde foi realizada uma massagem no sentido horário com duração de 15 minutos. O procedimento foi realizado nos dois primeiros dias do ciclo menstrual, ao longo de três ciclos (23).

A intensidade e duração das dores foram medidas por meio da escala VAS antes e após intervenção, em cada um dos ciclos. Os níveis de ansiedade foram medidos após 24 horas da massagem do segundo dia do ciclo, ao longo dos três ciclos (23), sendo avaliada pelo questionário de Spielberger $(24,25)$.

O Teste de Ansiedade de Spielberger consiste em um questionário de 25 itens e cada um destes é graduado em uma escala de 4 pontos: (nunca $=$ 0 , raramente $=1$, às vezes $=2$ e frequentemente $=3$ ). Nos itens do questionário estão inclusas sensações de tensão, preocupação, tranquilidade, proteção, dentre outros. Quanto maior a pontuação final obtida (somados os pontos dos 25 itens), maior o índice de estresse do participante $(24,25)$.

Com relação à intensidade da dor, a média pela escala VAS teve diminuição significativa entre o grupo experimental com lavanda e controle após o segundo e terceiro mês de intervenção $(\mathrm{P}<0,001)$. A respeito da duração das dores, após 
o terceiro mês de intervenção foi possível observar diminuição significativa da duração entre os grupos $(\mathrm{P}=0,006)$. Com relação aos níveis de ansiedade, os resultados do estudo não revelaram diferenças significativas entre os grupos de estudo em termos de níveis médios de ansiedade. No entanto, as comparações intragrupo indicaram uma significativa diminuição no nível de ansiedade do grupo de aromaterapia com lavanda após o terceiro ciclo $(\mathrm{P}=0.017)(23)$.

Quadro 2. Fase: Adulta/Reprodutiva da mulher e a utilização da aromaterapia utilizado óleos essenciais de diferentes espécies de Lavandula sp.

\begin{tabular}{|c|c|}
\hline $\begin{array}{c}\text { Autor: Yazdkhasti, M., Pirak A } \\
\text { Título: The Effect of Aromatherapy with Lavender Essence on Severity of } \\
\text { Labor Pain and Duration of Labor in Primiparous Women } \\
\text { Revista: Complementary Therapies in Clinical Practice } \\
\text { Ano: } 2016\end{array}$ & $\begin{array}{l}\text { Ensaio clínico com mulheres primíparas para avaliar se a aromaterapia } \\
\text { com L. angustifolia ameniza a intensidade e duração da dor durante o } \\
\text { parto. } \\
\qquad \mathrm{N} \text { amostral: } 120 \text { participantes }\end{array}$ \\
\hline $\begin{array}{c}\text { Autor: Kaviani M., Azima S., Alavi N., Tabaei M } \\
\text { Título: The effect of lavender aromatherapy on pain perception and } \\
\text { intrapartum outcome in primiparous women } \\
\text { Revista: British Journal of Midwifery } \\
\text { Ano: } 2014\end{array}$ & $\begin{array}{l}\text { Ensaio clínico com mulheres primíparas para avaliar se a aromaterapia } \\
\text { com } L \text {. angustifolia ameniza a intensidade da dor durante o parto, na } \\
\text { duração do trabalho de parto e na vitalidade do recém-nascido. } \\
\qquad \mathrm{N} \text { amostral: } 160 \text { participantes }\end{array}$ \\
\hline $\begin{array}{c}\text { Autor: Metawie M., Amasha H., Abdraboo R., Ali S } \\
\text { Título: Effectiveness of Aromatherapy with Lavender Oil in Relieving Post } \\
\text { Caesarean Incision Pain } \\
\text { Revista: Journal of Surgery } \\
\text { Ano: } 2015\end{array}$ & $\begin{array}{l}\text { Ensaio clínico com mulheres que passaram pelo parto cesárea para avaliar } \\
\text { o efeito da aromaterapia com Lavandula sp. na dor de incisão cesariana. } \\
\qquad \mathrm{N} \text { amostral: } 100 \text { participantes }\end{array}$ \\
\hline $\begin{array}{c}\text { Autor: Hadi, N., Hanid A } \\
\text { Título: Lavender essence for post-cesarean pain } \\
\text { Revista: Pakistan Journal of Biological Science } \\
\text { Ano: } 2011\end{array}$ & $\begin{array}{l}\text { Ensaio clínico com mulheres que passaram pelo parto cesárea para avaliar } \\
\text { o efeito da aromaterapia com L. angustifolia na dor pós-cesariana. } \\
\qquad \mathrm{N} \text { amostral: } 200 \text { participantes }\end{array}$ \\
\hline $\begin{array}{l}\text { Autor: Kianpour, M., Mansouri A., Mehrabi T., Asghari G } \\
\text { Título: Effect of lavender scent inhalation on prevention of stress, anxiety } \\
\text { and depression in the postpartum period } \\
\text { Revista: Indian } \\
\text { of Nursing and Midwifery Research } \\
\text { Ano: } 2016\end{array}$ & $\begin{array}{l}\text { Ensaio clínico com mulheres no período do pós-parto para avaliar o } \\
\text { efeito da aromaterapia com } L \text {. angustifolia na prevenção do estresse, } \\
\text { ansiedade e depressão pós-parto. } \\
\mathrm{N} \text { amostral: } 140 \text { participantes }\end{array}$ \\
\hline $\begin{array}{c}\text { Autor: Afhsar, M., Moghadam Z., Taghizadeh Z., Bekhradi R., } \\
\text { Montazeri A., Mokhtari P } \\
\text { Título: Lavender fragrance essential oil and the quality of sleep in } \\
\text { postpartum women } \\
\text { Revista: Iranian Red Crescent Medical Journal } \\
\text { Ano: } 2015\end{array}$ & $\begin{array}{l}\text { Ensaio clínico com mulheres no período do pós-parto para avaliar o } \\
\text { efeito da aromaterapia com Lavandula stoechas } L \text {. na qualidade do sono } \\
\text { neste período. } \\
\qquad N \text { amostral: } 158 \text { participantes }\end{array}$ \\
\hline $\begin{array}{l}\text { As análises realizadas e resultados encon- } \\
\text { trados em cada um dos estudos demonstram o } \\
\text { potencial da aromaterapia com óleos essenciais } \\
\text { de Lavandula sp., uma prática natural, acessível } \\
\text { e sem efeitos adversos para a diminuição e alívio } \\
\text { das dores do período menstrual, podendo ser uti- } \\
\text { lizada como um método capaz de trazer melhorias } \\
\text { no bem-estar e qualidade de vida de suas usuárias. } \\
\text { Os artigos apresentados no Quadro } 2 \text { discu- } \\
\text { tiram os benefícios da aromaterapia em sintomas } \\
\text { associados ao período reprodutivo e à gestação, } \\
\text { demonstrando os efeitos da Lavandula sp. na } \\
\text { intensidade e duração de dores de parto natural, } \\
\text { dores associadas à incisão em partos do tipo ce- }\end{array}$ & $\begin{array}{l}\text { sariano e em sintomas como estresse, ansiedade, } \\
\text { depressão e diminuição da qualidade de sono as- } \\
\text { sociados ao período do pós-parto. } \\
\text { O parto e o puerpério trazem inúmeros desa- } \\
\text { fios para as mulheres que passam por essa situa- } \\
\text { ção, vivenciando uma série de mudanças físicas } \\
\text { e mentais, desde a considerável diminuição das } \\
\text { horas de sono, até a perda de sua autonomia, tra- } \\
\text { zendo uma série de sentimentos novos que passam } \\
\text { a participar de suas tomadas de decisão (5), além } \\
\text { da possibilidade de desenvolvimento da depres- } \\
\text { são pós-parto, que possui etiologia multifatorial, } \\
\text { atingindo um número significativo de mulheres } \\
\text { durante esse período (6). }\end{array}$ \\
\hline
\end{tabular}


Os efeitos da aromaterapia com óleo essencial da L. angustifolia na dor e duração do trabalho de parto foram testados em um estudo onde 120 gestantes primíparas foram divididas em dois grupos: controle e experimental. As participantes do grupo experimental receberam gotas de uma solução (óleo essencial de lavanda diluído em uma proporção de 1:10 em água destilada) nas mãos e foram orientadas a esfregar as mãos e inalar a essência por 3 minutos a uma distância de 2,5-5 cm do rosto. O grupo controle foi tratado da mesma forma, porém apenas com água destilada. A intervenção foi realizada em 3 fases: dilatação 5-6-; 7-8; e 9-10 cm (26).

A intensidade da dor foi avaliada antes e 30 minutos após as três fases de intervenção, por meio da escala VAS. A duração das dores utilizou a mesma escala como método avaliativo. Para avaliar a vitalidade do recém-nascido, foi utilizada a pontuação de Apgar (26).

Com relação à intensidade da dor antes da intervenção, não foram observadas diferenças estatisticamente significativas entre os grupos $(\mathrm{P}=0,58)$. Após a intervenção, foram observadas diferenças estatisticamente significativas nas três fases avaliadas, ambas apresentando diminuição significativa da intensidade de dor do grupo experimental em comparação com o controle $(\mathrm{P}=0,001)(26)$.

Com relação à duração da fase ativa e à segunda fase do trabalho de parto, não foram observadas diferenças estatisticamente significativas entre os grupos, com os resultados de $(\mathrm{P}=0,5)$ e $(\mathrm{P}=0,6)$, respectivamente e por fim, com relação vitalidade do recém-nascido, não foram observadas diferenças estatisticamente significativas entre os grupos, com os resultados de $(\mathrm{P}=0,33)(26)$.

Ainda sobre as dores associadas ao parto, foi realizado um estudo com 160 gestantes primíparas que foram divididas em dois grupos: controle e experimental. As participantes do grupo experimental foram orientadas a manter panos de algodão contendo gotas de uma solução de óleo essencial de L. angustifolia diluído em água perto do rosto por alguns minutos. O grupo controle foi tratado da mesma forma, porém apenas com água destilada pingada na toalha (27).

A medida da intensidade/duração da dor foi realizada através da escala de VAS e ambos os grupos autoavaliaram seu nível de dor antes das intervenções e 30 e 60 minutos após elas. A pontuação de Apgar de neonatos no primeiro e quinto minutos após o nascimento da criança em ambos os grupos foram medidos e comparados (27).

Com relação à intensidade da dor, antes da intervenção não foram observadas diferenças estatisticamente significativas entre os grupos $(\mathrm{P}=0,286)$. Após a intervenção, foram observadas diferenças estatisticamente significativas 30 e 60 minutos após a intervenção - ambos com $(\mathrm{P}=0,001)(27)$.

Com relação à duração da fase ativa e à segunda fase do trabalho de parto, não foram observadas diferenças estatisticamente significativas entre os grupos e o mesmo ocorreu com a vitalidade do recém-nascido, com os resultados de $(\mathrm{P}=0,927)$ no $1^{\circ}$ minuto e de $(\mathrm{P}=0,749)$ no $5^{\circ}$ minuto após o nascimento (27).

Para avaliar a eficácia de aromaterapia com Lavandula sp. nas dores associadas à incisão do parto cesariana, foi realizado um estudo com 100 mulheres em pós-parto cesariano. As participantes foram divididas em dois grupos: controle e experimental. As mulheres de ambos os grupos foram submetidas ao tratamento de rotina do hospital como pós-medidas de alívio da dor pós-cesariana, e após seis horas (quando o efeito da anestesia tinha passado totalmente) o grupo experimental utilizou máscara facial de oxigênio durante três minutos (dentro da máscara foi aplicado $1 \mathrm{ml}$ de óleo essencial de Lavandula sp. - espécie não especificada) enquanto o grupo controle foi submetido ao mesmo procedimento, porém apenas com oxigênio no interior da máscara (28).

O nível de dor das participantes foi mensurado antes e após 30 minutos da intervenção, por meio da escala de VAS. Os resultados encontrados demonstraram que o grupo experimental obteve uma redução significativa de dores classificadas como severas, muito severas e pior possíveis quando comparadas ao grupo controle $(\mathrm{P}<0,001)$ (28).

Outro artigo que abordou a dor da incisão pós-cesariana como objeto de estudo foi realizado com 200 mulheres em pós-parto cesariano foram divididas em dois grupos - experimental e controle - e as participantes do primeiro grupo utilizaram máscara facial de oxigênio durante três 
minutos (dentro da máscara foram aplicadas 2 gotas de óleo essencial de L. angustifolia) enquanto o grupo controle foi submetido ao mesmo procedimento, porém com aroma sintético clinicamente neutro no interior da máscara (29).

O nível de dor das participantes foi autoavaliado por meio da escala VAS antes da intervenção (após mínimo de 3 horas desde o último recebimento analgésico intravenoso) e após 30 minutos, 8 horas e 16 horas da intervenção. Com relação à intensidade da dor antes da intervenção, não foram observadas diferenças estatisticamente significativas entre os grupos $(\mathrm{P}=0,18)$. Após a intervenção, houve diminuição dos níveis de dor nas três fases avaliadas, ambas apresentando resultado de $(\mathrm{P}<0,001)$ (29).

Visando verificar a eficácia da aromaterapia com Lavandula sp. em sintomas mentais do período do pós-parto, como estresse, ansiedade e depressão, foi realizado um estudo com 140 mulheres no período do pós-parto cesariano que foram divididas em dois grupos - experimental e controle - sendo as participantes do primeiro grupo submetidas à aromaterapia com óleo essencial de $L$. angustifolia, onde 3 gotas do óleo essencial foram colocadas em suas mãos, as mãos foram esfregadas e a essência inalada. $\mathrm{O}$ procedimento foi realizado $3 x$ por dia (a cada 8 horas) e durante 4 semanas após a alta do hospital. O grupo controle realizou o mesmo procedimento, porém com placebo (30).

Foram avaliados os níveis de estresse, ansiedade e depressão 2 semanas, 1 mês e 3 meses após a intervenção (30) e os métodos avaliativos utilizados foram a escala de Edimburgo (31) e a Escala de Depressão, Ansiedade e Stress (DASS) $(32,33)$.

A Escala de Depressão Pós-Parto de Edimburg (EPDS) consiste em um instrumento de autoavaliação composto por 10 itens referentes aos sintomas depressivos frequentemente observados no puerpério, como por exemplo, sentimento de culpa; distúrbios do sono; baixa energia; anedonia (perda da capacidade de sentir prazer, próprio dos estados gravemente depressivos), ideação suicida. A avaliação é realizada pela soma dos pontos de cada pergunta e pela adição das pontuações para cada um dos 10 itens. Quanto mais altos forem os índices, maiores os sintomas de depressão (31).
A escala de depressão, ansiedade e estresse (DASS) é capaz de medir e diferenciar os sintomas de ansiedade e depressão. Trata-se de um autorrelato composto por 21 questões, sendo dividido em 3 grupos de sintomas, contendo 7 perguntas em cada um desses grupos. São eles: (1) presença de afeto negativo, como humor deprimido, insônia, desconforto e irritabilidade, que são sintomas inespecíficos e estão incluídos tanto na depressão como na ansiedade; (2) fatores que constituem estruturas que representam sintomas específicos para depressão; (3) sintomas específicos de ansiedade (tensão somática e hiperatividade) $(32,33)$.

A pontuação é baseada em uma escala de quatro pontos, variando de 0 (não se aplicou a mim) a 3 (aplicou-se muito), referente ao sentimento da última semana. Soma-se o total de pontos de cada um dos grupos para obter-se a pontuação final. $(32,33)$.

Com relação aos resultados encontrados no estudo, houve diminuição significativa dos sintomas nas participantes do grupo experimental quando comparadas ao grupo controle já após duas semanas de intervenção (estresse $\mathrm{P}=0,012$ ); (ansiedade $\mathrm{P}=0,001)$ e (depressão $\mathrm{P}=0,003$ ). Após 1 e 3 meses de intervenção, também foram observadas diferenças significativas entre os grupos, com $(\mathrm{P}=0,001)$ para as três cadeias de sintomas testados (30).

Outro objeto de estudo da aromaterapia foi a qualidade do sono em mulheres no período pós-parto. Em ensaio clínico realizado com 158 mulheres no período do pós-parto foram divididas em dois grupos: experimental e controle (34).

O grupo experimental foi submetido à aromaterapia com óleo essencial de Lavandula stoechas a 10\%, diluído em óleo de gergelim. Foram aplicadas 4 gotas da mistura em uma bola de algodão e colocado em um recipiente cilíndrico, com uma distância projetada de $20 \mathrm{~cm}$. Elas inalaram por 10 respirações profundas e, em seguida, colocaram o recipiente ao lado de seu travesseiro até de manhã. O mesmo procedimento foi realizado com o grupo controle, porém apenas o óleo de gergelim foi utilizado no algodão. A intervenção foi realizada por 4 dias consecutivos na semana, durante 8 semanas. $O$ método utilizado para avaliar os sintomas das participantes foi o índice de qualidade do sono de Pittsburgh (34). 
O Índice da Qualidade do Sono de Pittsburgh (PSQI) avalia a qualidade subjetiva do sono, sendo um instrumento com confiabilidade e validade previamente estabelecidas. É um questionário composto por 19 itens, agrupados em sete componentes e cada um deles são pontuados 0 a 3. Os sete componentes são respectivamente: (1) a qualidade subjetiva do sono; (2) a latência do sono; (3) a duração do sono; (4) a eficiência habitual do sono; (5) as alterações do sono; (6) o uso de medicações para o sono; e (7) a disfunção diurna (35).

A pontuação obtida nos sete componentes é somada, gerando uma pontuação global do PSQI, podendo variar de 0 a 21 . Pontuações de 0-4 indicam boa qualidade do sono, de 5-10 indicam qualidade ruim e acima de 10 indicam distúrbio do sono (35).

Os resultados encontrados no estudo demonstraram que antes da intervenção, o índice de qualidade de sono não teve diferença significativa entre os grupos $(\mathrm{P}=0,64)$. Após 4 semanas de intervenção, obteve-se o valor $(\mathrm{P}=0,216)$ não comprovando diferenças entre os grupos e após 8 semanas de intervenção, houve a diminuição significativa dos sintomas nas participantes do grupo experimental quando comparadas ao grupo controle $(\mathrm{P}=0,033)$, demonstrando os benefícios da aromaterapia a longo prazo (34).

Quadro 3. Fase: Climatério e a utilização da aromaterapia utilizado óleos essenciais de diferentes espécies de Lavandula sp.

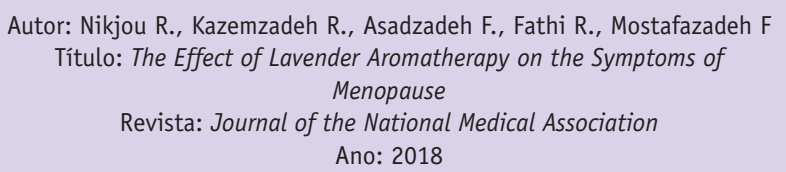

Autor: Kazemzadeh R., Nikjou R., Norouzi H., Rostamnegad M Título: Effect of lavender aromatherapy on menopause hot flushing: A crossover randomized clinical trial

Revista: Journal of the Chinese Medical Association Ano: 2016
Ensaio clínico com mulheres na menopausa para avaliar se a aromaterapia com Lavandula sp. diminuiu a intensidade dos sintomas do climatério. $\mathrm{N}$ amostral: 100 participantes

Realiza ensaio clínico com mulheres na menopausa para avaliar se a aromaterapia com Lavandula sp. traz melhoria ao sintoma de rubor característico do climatério. $\mathrm{N}$ amostral: 100 participantes
Os artigos apresentados no Quadro 3 discorrem sobre os benefícios da aromaterapia com $L a$ vandula sp. em sintomas associados ao período do climatério, marcado por desafios e mudanças nas vidas das mulheres, com associação entre sintomas climatéricos intensos e o desenvolvimento de sintomas de depressão e ansiedade, além da auto percepção de estado de saúde ruim ou péssima, causada pelo efeito prejudicial de um pior estado psicológico (7).

Em um estudo, 100 mulheres no período da menopausa foram divididas em dois grupos experimental e controle - para avaliar os efeitos da lavanda nos sintomas climatéricos. O grupo experimental foi submetido à aromaterapia com óleo essencial de Lavandula sp. onde o óleo foi pingado dentro de um copo e inalado pelas participantes por 20 minutos, duas vezes por semana, ao longo de doze semanas. O grupo controle foi submetido ao mesmo procedimento, porém com leite diluído usado como placebo. A autoavaliação dos sintomas pelas participantes foi realizada através da Escala Climatérica de Greene antes e ao final da intervenção (36).

A Escala Climatérica de Greene é utilizada para avaliar a presença e intensidade dos sintomas climatéricos. É composta por 21 perguntas, cada uma delas avaliada por uma escala ordinal pontuada de 1 a 4, onde: 1 representa "ausência de sintomas"; 2 "sintomas leves"; 3 "sintomas moderados" e 4 "sintomas intensos". A escala de Greene também é dividida em sub escalas, como a psicológica (dividida em ansiedade e depressão), a somática e a de disfunção sexual. A soma da pontuação total das 21 questões e seus aumentos/ reduções ao longo de qualquer tratamento repre- 
senta a melhora ou piora clínica dos sintomas climatéricos (37).

Os resultados encontrados no estudo demonstraram que não houve diferença significativa entre os grupos para os 5 sintomas avaliados (ansiedade, depressão, sintomas físicos, sintomas vasomotores e desejo sexual) antes da intervenção $(\mathrm{P}>0,05)$. Após o período completo de intervenção, houve diminuição estatisticamente significativa dos sintomas para as participantes do grupo experimental quando comparados ao grupo controle, com valor $(\mathrm{P}<0,001)$ para os 5 grupos de sintomas autoavaliados (36).

Como ponto inicial da discussão, é importante enfatizar que a proposta do trabalho foi verificar se a aromaterapia com óleo essencial de Lavandula sp. é capaz de amenizar sintomas vividos pelas mulheres por si só, trazendo melhoria na qualidade de vida das mesmas sem intervenção medicamentosa.

Os estudos clínicos escolhidos para o desenvolvimento do trabalho que foram apresentados nos Quadros 1 a 3 não estabelecem comparação entre as respostas clínicas de pacientes com utilização de terapia medicamentosa convencional e aromaterapia com óleos essenciais de Lavandula sp., fato estabelecido nos critérios de inclusão e exclusão dos artigos selecionados, onde pacientes que faziam a utilização de qualquer tipo de medicamento para tratar o sintoma discutido naquele artigo, não foram consideradas aptas para participarem do ensaio clínico.

Os artigos selecionados utilizaram como base estatística o valor de $\mathrm{P}$, onde dados obtidos com valor de $\mathrm{P}<0,05$ foram considerados estatisticamente significativos, comprovando as diferenças no estado clínico das participantes do estudo antes e após a intervenção com a aromaterapia.

Para compreender os resultados obtidos nos artigos selecionados, é importante mencionar qual o mecanismo de ação do óleo essencial de lavanda no sistema nervoso e como ele atua no organismo humano.

A Lavandula sp. pode ser encontrada em diversas espécies, mas a principal delas e a mais frequentemente utilizada para produção dos óleos essenciais na aromaterapia é a L. angustifolia. To- das as espécies possuem os mesmos metabolitos secundários principais, como o linalol, acetato de linalil, 1,8-cineol $\beta$-ocimeno, terpinen-4-ol e cânfora, variando apenas o nível relativo de cada um desses constituintes de acordo com a espécie (38).

De acordo com a Farmacopeia Europeia, o linalol e acetato de linalila são os principais compostos responsáveis pelos efeitos terapêuticos da aromaterapia com Lavandula sp, representando, respectivamente, $(20-50 \%)$ e $(25-46 \%)$ da composição do óleo essencial de lavanda da espécie $L$. angustifolia (15).

O processo olfativo constitui uma série de reações interligadas, químicas e elétricas. As partículas voláteis desprendidas do material odorante são carregadas pelo ar e atingem a cavidade nasal, onde se encontram as células receptoras olfativas. Esses receptores dão origem à vesícula olfatória que, por sua vez, originam os cílios sensoriais responsivos aos estímulos químicos (14).

Nessa região, existem proteínas fixadoras de odoríferos, responsáveis pela transdução olfativa (conversão de um sinal químico em elétrico). Impulsos nervosos são desencadeados e encaminhados ao bulbo olfatório e, por meio dos glomérulos ali presentes, esses impulsos são direcionados ao sistema nervoso central, alcançando também o sistema límbico e suas estruturas tais como a amígdala, tálamo e hipotálamo. Os compostos voláteis podem ainda entrar na corrente sanguínea por meio da mucosa pulmonar (14).

Os mecanismos de ação do linalol e do acetato de linalila no tratamento de ansiedade, estresse, depressão e dores de diversas causas ainda não são completamente elucidados $(39,40)$.

Com relação à atividade ansiolítica do óleo essencial de lavanda, estudos indicam que esse efeito se dá pela redução da atividade do receptor 5HT1A (receptor serotoninérgico acoplado à proteína Gi e de caráter inibitório) aumentando assim a atividade da serotonina, e pelo aumento do tônus parassimpático em detrimento do simpático (principal sistema ativado em casos de estresse e ansiedade) (39).

Existem também pesquisas que sugerem que essas substâncias são capazes de inibir o metabolismo das catecolaminas, diminuindo o estímulo 
de estresse ou medo enviado ao hipotálamo e provocando, indiretamente, uma redução nos níveis do hormônio adrenocorticotrófico e consequentemente na produção de cortisol pela glândula adrenal (39).

Estudos indicam ainda, que os impulsos nervosos, que chegam ao sistema límbico por meio da via olfativa, estimulam a liberação de neurotransmissores como a endorfina, encefalina e a serotonina, associados à sensação de bem-estar. Sabe-se que uma das possíveis causas da depressão é a diminuição dos níveis de serotonina, justificando os benefícios da aromaterapia com lavanda em pacientes que sofrem desta desordem (41).

Com relação ao potencial analgésico do óleo essencial de lavanda, estudos demonstram que este pode ser proporcionado em decorrência da atuação do linalol como um antagonista competitivo dos receptores NMDA, receptor glutamatérgico presente nas fibras nervosas e sistema nervoso central, fundamental na sinalização da dor (42).

\section{CONCLUSÃO}

Por meio dos resultados obtidos e da discussão gerada, foi possível observar que os ensaios clínicos realizados demonstraram efeitos positivos com relação à aromaterapia com diferentes espécies de Lavandula sp., em especial a L. angustifolia. As dores associadas à dismenorreia, ao trabalho de parto e pós-parto cesariano foram reduzidas com significância estatística nas mulheres que foram submetidas ao procedimento controle em comparação ao grupo placebo. O mesmo aconteceu com sintomas mentais associados ao pós-parto e climatério. Ainda que a aromaterapia não tenha sido eficaz para alívio de alguns grupos de sintomas, como a duração da dor de parto ou o volume de sangramento menstrual, e que, tenha apenas amenizado os sintomas onde se mostrou eficaz, essa terapia se mostrou bastante positiva como um todo, tendo em vista, além dos os resultados satisfatórios dos estudos clínicos, seu custo reduzido e acessível, sua ausência de eventos adversos e seu vínculo ao SUS, enquanto uma prática integrativa e complementar à saúde.

\section{REFERÊNCIAS}

1. Zampieri MD, Tavares CM, Hames MD, Falcon GS, Silva AL, Gonçalves LT. O processo de viver e ser saudável das mulheres no climatério. Esc. Anna Nery. 2009;13(2):305312. DOI: $10.1590 / \mathrm{s} 1414-81452009000200010$

2. Frare JC, Tomadon A, Silva JR. Dismenorreia: Prevalência e efeito na qualidade de vida. RBCS. 2014;12(39):15-20. DOI: 10.13037/rbcs.vol12n39.2095

3. Muramatsu CH, Vieira OC, Simões CC, Katayama DA, Nakagawa FH. Consequências da síndrome da tensão pré-menstrual na vida da mulher. Rev. Esc. Enferm .2001;35(3):205-213. DOI: 10.1590/s008062342001000300002

4. Rapoport A, Piccinini CA. Apoio social e experiência da maternidade. Rev Bras Cresc Desenv Humano. 2006;16(1):85. DOI: 10.7322/jhgd.19783

5. Souza BMS, Souza SF, Rodrigues RTS. O puerpério e a mulher contemporânea: uma investigação sobre a vivência e os impactos da perda da autonomia. Rev. SBPH. 2013;16(1):166-184. Disponível em: http://pepsic. bvsalud.org/scielo.php?script=sci_arttext\&pid=S151608582013000100010\&lng=pt.
6. Schmidt EB, Piccoloto NM, Müller MC. Depressão pós-parto: fatores de risco e repercussões no desenvolvimento infantil. Psico-USF. 2005;10(1):61-68. DOI: 10.1590/s141382712005000100008

7. Lui Filho JF, Baccaro LF, Fernandes T, Conde DM, CostaPaiva L, Pinto Neto AM. Epidemiologia da menopausa e dos sintomas climatéricos em mulheres de uma região metropolitana no sudeste do Brasil: inquérito populacional domiciliar. Rev. Bras. Ginecol. Obstet. 2015;37(4):152-158. DOI: $10.1590 /$ so100-720320150005282

8. BRASIL. Práticas integrativas e complementares na saúde PICS. Conselho Federal de Fisioterapia e Terapia Ocupacional. Disponível em: https://coffito.gov.br/ campanha/pics/index.php?nome=principal\#geral.

9. BRASIL. Práticas integrativas e complementares (PICS): quais são e para que servem. Ministério da Saúde. 2019. Disponível em: https://saude.gov.br/saude-de-a-z/praticasintegrativas-e-complementares

10. BRASIL. Práticas integrativas e complementares - PICs. UFJF, 2009. Disponível em: https://ufjf.br/integralidade/ saber-mais/pic/. 
11. BRASIL. Portaria $n^{\circ} 702$, de 21 de março de 2018: Altera a Portaria de Consolidação n ${ }^{0}$ 2/GM/MS, de 28 de setembro de 2017, para incluir novas práticas na Política Nacional de Práticas Integrativas e Complementares - PNPIC. Ministério da Saúde. Diário Oficial da União, nº702, 22 de março de 2018. Seção 1. p. 65. Disponível em: http://bvsms.saude.gov.br/bvs/saudelegis/gm/2018/ prt0702_22_03_2018.html

12. Gnatta JR, Kurebayashi LF, Turrini RN, Silva MJ. Aromatherapy and nursing: historical and theoretical conception. Rev Esc Enferm USP. 2016;50(1):127-133. DOI: $10.1590 / \mathrm{s} 0080-623420160000100017$

13. BRASIL. Sobre a Abraroma. Abraroma, 2018. Disponível em: https://aromaterapia.org.br/sobre/.

14. Andrei P, Comune APD. Aromaterapia e suas aplicações. Centro Universitário S. Camilo. 2005;11(4):57-68. Disponível em: http://www.saocamilo-sp.br/pdf/ cadernos/36/07_aromaterapia.pdf.

15. Alves, B. Óleo essencial de lavanda (Lavandula angustifolia) no tratamento da ansiedade. [Tese]. São João del Rei. Universidade Federal de São João del-Rei, 2018. Disponível em: https:/www.ufsj.edu.br/portal2-repositorio/File/coqui/ TCC/Monografia-TCC-Barbara.pdf

16. Gören AC, Topçu G, Bilsel G, Bilsel M, Aydoğmusç Z, Pezzuto JM. The Chemical Constituents and Biological Activity of Essential Oil of Lavandula stoechas ssp. stoechas. Z Naturforsch C J Biosci, 2002 ;57(9-10):797800. DOI: $10.1515 /$ znc-2002-9-1007

17. Neuwirth A, Chaves ALR, Bettega JMR. Propriedade dos óleos essenciais de cipreste, lavanda e hortelã-pimenta. Artigo Científico UNIVALE, 2020. Disponível em: https:// sapiens.univali.br/propriedades-dos-oleos-essenciais-decipreste-lavanda-e-hortela-pimenta

18. BRASIL. Cólicas menstruais. Ministério da Saúde. 2012. Disponível em: https://bvsms.saude.gov.br/bvs/ dicas/252_colicas_menstruais.html

19. Karimollahi M, Nikjou R, Kazemzadeh R, Rostamnegad M, Moshfegi S, Salehi H. The effect of lavender aromatherapy on the pain severity of primary dysmenorrhea: A tripleblind randomized clinical trial. Ann Med Health Sci Res. 2016;6(4):211. DOI: 10.4103/amhsr.amhsr_527_14

20. USA. Visual Analog Scale. Painscale. Disponível em: https://www.painscale.com/article/visualanalog-scal

21. Dehkordi ZR, Baharanchi FSH, Bekhradi R. Effect of lavender inhalation on the symptoms of primary dysmenorrhea and the amount of menstrual bleeding: A randomized clinical trial. Complement Ther Med. 2014;22(2):212-219. DOI: 10.1016/j.ctim.2013.12.011

22. Zayeri F, Dehkordi ZR, Hosseini-Baharanchi FS. The clinical efficacy of lavender oil inhalation on intensity of menstrual pain from primary dysmenorrhea. J Herbmed Pharmacol. 2019; 8(3):218-223. DOI: 10.15171/ jhp.2019.32

23. Azima S, Bakhshayesh HR, Kaviani M, Abbasnia K, Sayadi M. Comparison of the Effect of Massage Therapy and Isometric Exercises on Primary Dysmenorrhea: A Randomized Controlled Clinical Trial. J Pediatr Adolesc Gynecol. 2015;28(6):486-491. DOI: 10.1016/j. jpag.2015.02.003

24. Bekhradi R, Vakilian K. The effect of lavender aromatherapy on test anxiety in female students. Curr. Women's Health Rev. 2017;12(2):137-140. DOI: 10.217 $4 / 1573404812666161021114923$

25. USA. The state-trait anxiety inventory (STAI). American Psychological Association. 2011. Disponível em: https:// www.apa.org/pi/about/publications/caregivers/practicesettings/assessment/tools/trait-state

26. Yazdkhasti M, Pirak A. The effect of aromatherapy with lavender essence on severity of labor pain and duration of labor in primiparous women. Complement Ther Clin Pract. 2016; 25:81-86. DOI: 10.1016/j.ctcp.2016.08.008

27. Kaviani M, Azima S, Alavi N, Tabaei MH. The effect of lavender aromatherapy on pain perception and intrapartum outcome in primiparous women. Br. J. Midwifery. 2014;22(2):125-128. DOI: 10.12968/bjom.2014.22.2.125

28. Metawie MA-H. Effectiveness of Aromatherapy with Lavender Oil in Relieving Post Caesarean Incision Pain. Journal of Surgery. 2015;3(2):8. DOI: 10.11648/j. js.s.2015030201.12

29. Hadi N, Hanid AA. Lavender Essence for Post-cesarean Pain. Pak. J. Biol. Sci. 2011;14(11):664-7. DOI: 10.3923/ pjbs.2011.664.667

30. Kianpour M, Mansouri A, Mehrabi T, Asghari G. Effect of lavender scent inhalation on prevention of stress, anxiety and depression in the postpartum period. Iran. J. Nurs. Midwifery Res. 2016;21(2):197. DOI:10.4103/17359066.178248

31. Malloy-Diniz LF, Schlottfeldt CG, Figueira P, Neves FS, Corrêa H. Escala de Depressão Pós-Parto de Edimburg: análise fatorial e desenvolvimento de uma versão de seis itens. Braz J Psychiatry. 2010;32(3):316-318. DOI:10.1590/ s1516-44462010000300018

32. Corrêa CA, Verlengia R, Ribeiro AG, Crisp AH. Níveis de estresse, ansiedade, depressão e fatores associados durante a pandemia de COVID-19 em praticantes de Yoga. Bras Ativ Fís Saúde. 2020;25:1-7. DOI:10.12820/rbafs.25e0118

33. Fonseca DC, Galdino DA, GuimarãesLH, AlvesDA. Avaliação da qualidade do sono e sonolência excessiva diurna em mulheres idosas com incontinência urinária. Revista Neurociências. 2001;18(3):294-299. DOI:10.34024/r nc.2010.v18.8458 
34. Afshar MK, Moghadam ZB, Taghizadeh Z, Bekhradi R, Montazeri A, Mokhtari P. Lavender Fragrance Essential Oil and the Quality of Sleep in Postpartum Women. . Iran Red Crescent Med J. 2015;17(4). DOI:10.5812/ ircmj.17(4)2015.25880

35. Montgomery M, Marinho R, Santiago RC, Surita R. Avaliação do efeito da tibolona na sexualidade de mulheres na pós-menopausa. Reprod. Clim. 2000;15(1):28-34.

36. Nikjou R, Kazemzadeh R, Asadzadeh F, Fathi R, Mostafazadeh F. The effect of lavender aromatherapy on the symptoms of menopause. J Natl Med Assoc. 2018;110(3):265-269. DOI:10.1016/j.jnma.2017.06.010

37. Montgomery M, Marinho R, Santiago RC, Surita R. Avaliação do efeito da tibolona na sexualidade de mulheres na pós-menopausa. Reprod. Clim. 2000;15(1):28-34.

38. Koulivand PH, Ghadiri MK, Gorji A. Lavender and the Nervous System. Evid Based Complement Alternat Med. 2013;1-10. DOI:10.1155/2013/681304
39. Lima LKS. Efeito agudo do óleo essencial de lavandula angustifolia nos níveis de craving e ansiedade em usuário de crack. [Tese]. João Pessoa, Universidade Federal da Paraíba, 2019.

40. Nikfarjam M, Parvin N, Assarzadegan N, Asghari S. The Effects of Lavandula angustifolia mill Infusion on Depression in Patients Using Citalopram: A comparison Study. Iran Red Crescent Med J. 2013;15(8):734-739. DOI:10.5812/ircmj.4173

41. Patias ND, Machado WDL, Bandeira DR, Dell'Aglio DD. Depression Anxiety and Stress Scale (DASS-21) - Short Form: Adaptação e Validação para Adolescentes Brasileiros. Psico-USF. 2016;21(3):459-469. DOI: 10.1590/141382712016210302

42. Peana AT, D'Aquila PS, Chessa MLoredana, Moretti MDL, Serra G, Pippia P. (-)-Linalool produces antinociception in two experimental models of pain. Eur J Pharmacol. 2003;460(1):37-41. DOI: 10.1016/S00142999(02)02856-X 